\title{
Feasibility and outcomes from development and testing of a health behavior theory-based fitness app
}

\author{
Lynn Katherine Herrmann ${ }^{1}$, Sarah R. Blackstone ${ }^{2}$ \\ ${ }^{1}$ School of Health Studies - Public Health and Health Education, Northern Illinois University, DeKalb, IL, USA; ${ }^{2}$ Department of Health Sciences, \\ James Madison University, Harrisonburg, VA, USA \\ Correspondence to: Lynn Katherine Herrmann, PhD, MPH. School of Health Studies - Public Health and Health Education, Northern Illinois \\ University, 370 Wirtz Drive, Wirtz Hall 209, DeKalb, IL 60115, USA. Email: LHerrmann@niu.edu.
}

\begin{abstract}
Research studies suggest that health behavior theory-informed approaches in behavior change interventions are needed to evoke improved health outcomes. This is specifically noted in mobile fitness applications ("apps"), for which the literature indicates a gap between theory use and app development. The purpose of this study was to develop and test a theory-based app to determine if various constructs, in the form of app features, could be manipulated to elicit behavior change. The hypothesis was that the version of the app that included all constructs of TPB would be most impactful at increasing behavioral intention and the actual behavior of engaging in more physical activity. A fitness app was developed with Theory of Planned Behavior (TPB) constructs used to inform app features. The app basic features included behavior tracking, pre-programmed workouts, and a user-friendly interface. The app had seven variations with certain app features, designed to address TPB constructs, available or not available, to the user. After completing the pre-test, 53 participants received a version of the app at random and were not aware of other possible app version features. After eight weeks of using the app, participants completed a post-test (N=44). Preand post-test surveys measured TPB constructs. The participants were primarily white women ages 18-24. Due low enrollment and attrition, control participants were removed from the analysis and all versions of the app were collapsed into one group for pre- post-test comparison. Between pre- and post-test, across all app versions with features addressing TPB constructs, "intention to exercise using a fitness app" increased significantly $[\mathrm{t}(40)=3.88, \mathrm{P}<0.001]$. However, overall "exercise intention" and actual reported exercise frequency did not change. Although the theory-based app did evoke change in behavioral intention with regard to using a fitness app, this did not translate to reported increases in exercise frequency. Though the app incorporated a health behavior change theory, the app did not elicit behavior change, which may be due to sample size or other factors that have yet to be elucidated. There is much room for improvement in fitness app development, such as translating intention to behavior, testing other health behavior theories, and determining the best ways to develop app features to represent theoretical constructs.
\end{abstract}

Keywords: Fitness app; development; outcomes; Theory of Planned Behavior (TPB)

Received: 03 March 2020; Accepted: 14 July 2020; Published: 31 July 2020.

doi: $10.21037 /$ ht-20-17

View this article at: http://dx.doi.org/10.21037/ht-20-17

\section{Introduction}

Fitness applications (apps) have become increasingly popular over the last decade as a way to set fitness goals, track activity and share progress toward fitness goals $(1,2)$. As use of apps continues to increase, studies have examined the relationship between use of apps and subsequent fitness behaviors with mixed results. One benefit is that fitness tracking over time allows users to see trends in their own behavior, which can have a positive impact on health (3). However, in examining adherence and effectiveness of fitness apps over five months, one study found that personal 
fitness perceptions and exercise behavior did not change from pre- to post-test (4). The only significant change was a decrease in perceived usefulness of using apps for exercising from baseline to posttest.

One issue that has been identified with fitness apps, and may contribute to their limited effectiveness overtime, is the lack of theory-based apps (5). Many apps do not use health behaviors theories to guide their initial development; if theories are used, not all the constructs are applied, making it challenging to determine efficacy of theory application $(6,7)$. An examination of 23 health apps with high consumer reviews in online app stores determined that all the apps lacked theory-based behavior change strategies (8). Another study reviewing 127 apps from an app store's Health and Fitness category also indicated a general lack of application of theory-based behavior change strategies (5). Even the presence of behavior change techniques is quite low among popular apps. One study found that most of the top ranked health and fitness apps used fewer than four behavior change techniques, the most common being instruction on exercise execution, modeling of exercises, feedback on performance, activity goal setting, and planning for social support and behavior change (9). Another study found that the presence of behavior change strategies were included in the paid apps and not in the free apps, which brings about concerns for accessibility and affordability (10). The use of behavior change techniques may be a moot point as there is lack of consensus if behavior change techniques alone can change behavior $(11,12)$; instead, the use of full behavior change theories with all the theory constructs may be a better avenue to behavior change.

Theory based interventions are known to be effective in initiating and sustaining health behavior change (13), yet the majority of available apps for fitness and health do not have theoretical foundations in behavior change. While this is not unexpected, as app developers' expertise is in software development and may not include health behavior theory, utilizing health behavior theory in app development can provide more compelling evidence of apps' effectiveness (or lack of) in sustaining behavior change (14). It has been suggested that input from health behavior change specialists could mitigate some of these noted limitations (14). In the meantime, assessment tools for apps have been developed to help professionals determine appropriateness of apps for clients, such as the Mobile App Rating Scale (15).

It can be posited that app effectiveness could be driven not only by user interface and usability, but also the inclusion of health behavior change theory. That said, the purpose of this pilot study was to investigate the use of a health behavior theory in development and evaluation a fitness app. The original hypothesis was that the version of the app that included all constructs of TPB would be most impactful at increasing behavioral intention and the behavior of engaging in more physical activity. Examining the use of theory in development of a fitness app could provide insight into the feasibility of incorporating theory constructs into the app and understanding related outcomes.

\section{Methods}

\section{Theory selection}

In order to begin the process of understanding how health behavior theory could be used to inform development of a fitness app, an appropriate theory was selected. There are several health behavior theories that have been examined for exercise behavior change, including the Transtheoretical Model, the Health Belief Model, and the Theory of Planned Behavior (TPB). The Transtheoretical Model was rejected due to the focus on readiness for change and weighing pros and cons. These areas might help determine if the user would be ready to use the app, but not actively help understand the use of the app. The Health Belief Model was rejected due to the focus on perceptions of engaging or not engaging in a behavior, which would not help the understanding of actual behavioral engagement of the app. Instead, TPB was selected for the development of this app because the constructs of attitude, subjective norms, and perceived behavior control, can be manipulated to influence behavior and been well studied in relation to exercise behavior in both apparently healthy populations and populations with disease (16). Additionally, there is an already existing validated TPB survey related to exercise behavior (17), which was adapted to include exercise using a fitness app. The following TPB constructs were measured in the survey. Attitude is one's beliefs about a behavior and the expected outcomes from engaging in the behavior. Subjective norms relate to behaviors one considers to be "normal" or typical and the extent to which one is willing to comply to the norm. Perceived behavior control regards the extent to which one thinks they have control over the behavior and how much these beliefs can be influenced. Lastly, behavior intention is one's likelihood of engaging in the behavior. As noted in TPB, behavioral intention does not necessarily lead to the actual behavior.

Perhaps most importantly when selecting an appropriate 
Table 1 Theory constructs mapped to app features

\begin{tabular}{lll}
\hline Theory construct & Area of potential change & App feature \\
\hline Attitude & $\begin{array}{l}\text { Knowledge, expectation of } \\
\text { outcomes }\end{array}$ & $\begin{array}{l}\text { Modeling of exercise to influence attitude, knowledge, and outcome } \\
\text { expectations }\end{array}$ \\
Subjective norms & $\begin{array}{l}\text { Motivation to behave a certain } \\
\text { way }\end{array}$ & $\begin{array}{l}\text { Cues to action to influence beliefs on what is significant, motivate to comply } \\
\text { with the norm } \\
\text { Perceived behavioral } \\
\text { control }\end{array}$ \\
\hline
\end{tabular}

theory from an app development perspective, TPB constructs could be translated into app features. The process of mapping the theory constructs to app features involved examining what could be measured within the construct and how that measure could be translated into an app feature. For the construct of attitude, modeling of exercises through photos, not just text descriptions of exercise, has been linked to increased exercise behaviors (18). For the construct of subjective norms, the motivation to comply with the notification was mapped to how the notification (or cue to action) influenced the user's beliefs on what was important and the extent to which the user was motivated to comply with the notification. Previous research shows that app notifications can motivate behavior change (19). For the construct of perceived behavioral control, a person's ability to believe they can accomplish a behavior and overcome potential barriers is related to self-efficacy. Self-efficacy has been linked to goal setting and goal achievement (20). App features and design are discussed further in the next section, including Table 1, which summarizes the mapping of constructs to app features.

\section{App feature development in relation to TPB}

Initially, the research team compiled a list of potential app features that relate to TPB constructs. This list was brought to the app development team at one researcher's university to determine the feasibility of building the app features for an app on Android devices. Only Android devices were included in this study due to the more open standards for app development (no required specifications by the app store) and no cost to publish the app in the app store. The app development team then developed the app, which was tested by the research team to check for and correct errors before the app was released to study participants.

The most basic version of the fitness app features included behavior tracking, pre-programmed workouts, and a user-friendly interface. None of these basic app features related to TPB constructs. For behavior tracking, users logged activity by selecting from a list of exercise and sport types. For pre-programmed workouts, the user received text (typed) instructions to follow along with a workout designed by a certified fitness professional. The seven preprogrammed workouts included: Sunrise Stretch (yoga for the morning), Deskercise (exercise that could be performed while seated or at a desk), High Intensity Interval Training, Lower Body Strength, Upper Body Strength, Core Stability and Strength, and Evening Stretch (yoga for before bedtime). Lastly, the interface was based on best practices for app development, such as data privacy and minimal menus $(1,21)$.

Features of app variation included cues to action, goalsetting function, and modeling (photos) of exercises in pre-programmed workouts. Cues to actions included text reminders to move and follow goals. The goal-setting function allowed users to select type of activity, minutes of the activity, and date to complete the goal. Modeling (photos) of exercise in pre-programmed workouts provided a visual to match the text (typed) instructions for each workout. All app features needed to be manually accessed by the user aside for the cues to action, which was sent as a notification to the user's device. In TPB, these three constructs lead to behavioral intention, which then, in theory, leads to behavior change. Figure 1 shows several of the screens within the app.

The following table shows the theory construct, the area of potential change, and the associated app features.

\section{Recruitment}

After obtaining IRB approval, study participants were recruited through one of the researcher's university email newsletter, which came out twice weekly over a onemonth timeframe. The advertisement only said the study 

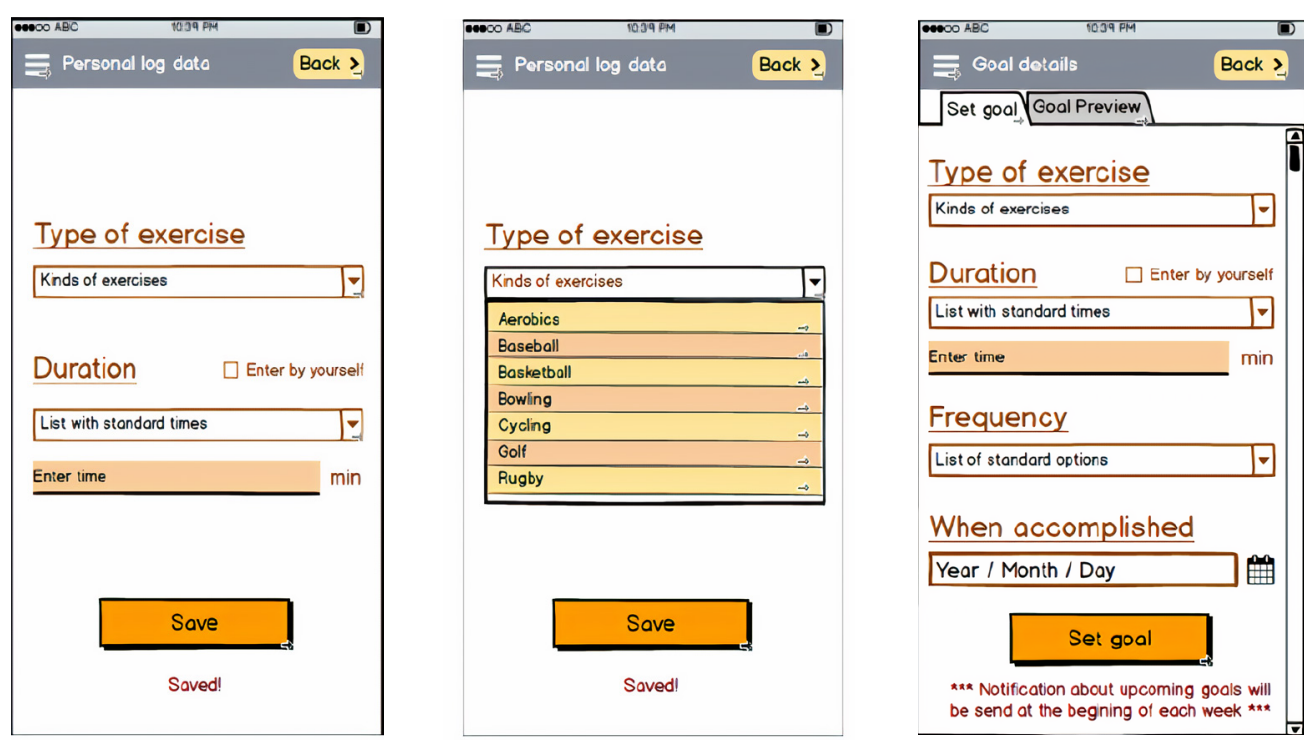

Figure 1 Examples of app features.

was recruiting individuals over the age of 18 , who own an Android device, and are interested in testing out a fitness app. Per the study advertisement, interested individuals emailed the researcher to get directions on how to take the online pre-test via Qualtrics using their participant identification number, fill out the online consent form, and download a randomized version of the app. The research team was aware of the app version the participant received. Participants were told to use the app over an eight-week timeframe however they would like; they were not given any app usage requirements. At the end of the timeframe, they would receive an email with the post-test via Qualtrics using the participant identification number. All prospective participants who emailed the researcher with interest in participating in the study went on to take the pretest and download the app.

\section{App variations}

The app had seven variations with certain app features related to TPB available or not available to the user. All apps included the basic features (described above in "App feature development in relation to TPB"). Participants were not aware of the version of their app or the features they did or did not have. They were also not made aware that certain app features related to constructs of TPB.

\section{Assessment}

Pre- and post-test surveys measured different constructs related to TPB. The validated survey (17) was used to assess attitudes, perceived behavioral control, subjective norms and exercise intention to exercise general and exercise specifically using a fitness mobile application. Attitudes were assessed using seven questions regarding attitudes toward exercise (range, 7-49; $\alpha=0.94$ ). Subjective norms were measured using two questions ascertaining behaviors of those close to the individual (range, $7-14 ; \alpha=0.87$ ). Perceived behavioral control was assessed using three questions measuring an individual's perception of their ability to engage in exercise (range, $7-21 ; \alpha=0.88$ ). Finally, behavioral intention was measured with a single item question asking how frequently the participant planned on exercising in the next week (range, 1-9). Additional questions included perception of personal fitness and comfort level of using and learning new technology. Demographic information including age, gender, education, and employment was obtained at pre-test. Paired $t$-tests were used to examine if significant changes in attitudes, subjective norms, perceived behavioral control, and behavioral intentions occurred between pre- and post-test.

\section{Results}

Initially, 53 participants completed the pre-test and were assigned to one of the seven app versions or the control group. Eleven participants did not complete the study. At the end of the study, four participants assigned the control version of the app completed both pre- and post-test. The 
Table 2 Summary of app features

\begin{tabular}{lccccc}
\hline App \# & Modeling of exercise & Cues to action & Goal setting & Pre-test $(\mathrm{n})$ & Post-test $(\mathrm{n})$ \\
\hline App 1 & On & On & On & 5 & 3 \\
App 2 & On & On & Off & 6 & 5 \\
App 3 & On & Off & On & 7 & 6 \\
App 4 & On & Off & Off & 7 & 6 \\
App 5 & Off & On & On & 6 & 5 \\
App 6 & Off & On & Off & 8 & 6 \\
App 7 & Off & Off & On & 7 & 6 \\
App 8 & Off & Off & Off & 7 & 4 \\
\hline
\end{tabular}

Table 3 Pre-post-test for all participants who received the app versions with TPB constructs

\begin{tabular}{lcc}
\hline Variable & Mean difference & t statistic \\
\hline Exercise frequency & 0.561 & 1.795 \\
Exercise intention & 0.098 & 0.474 \\
Exercise intention using a fitness app & 0.732 & 3.889 \\
Technology-use & 0.108 & 0.344 \\
Technology-learning new & 0.081 & 0.261 \\
Exercise attitude & 0.231 & 0.144 \\
Exercise attitude regarding using fitness app & -2.395 & -1.314 \\
Subjective norms regarding exercise & -0.250 & -0.383 \\
Subjective norms regarding exercise using fitness app & 0.250 & 0.733 \\
Perceived control over exercise & 0.415 & 0.832 \\
Perceived control over exercise using fitness app & 1.725 & 0.197 \\
Perception of fitness & -0.735 & 0.704 \\
\hline
\end{tabular}

*, significant at $P \leq 0.001$.

other participants were assigned a version of the app with at least one TPB construct, but potentially all TPB constructs (see Table 2).

Of the 44 participants who completed the post-test, the majority $(\mathrm{n}=32,78 \%)$ of participants were white women. About half $(53 \%)$ of the sample indicated their age was within the 18-24 range. Due to small sample size and limited participants receiving each version of the app, meaningful group comparison between control group and app versions was not possible. Therefore, all participants who received any app variation were combined into one group, and four participants who received the control condition were removed from the analysis, bringing the total sample used in the final analysis to 41 . Between preand post-test, across all app versions with TPB constructs, intention to exercise using a fitness app increased significantly $\mathrm{t}(40)=3.88, \mathrm{P}<0.001$. However, overall exercise intention and actual exercise frequency did not change. Attitude, subjective norms, and perceived behavior control had no significant difference pre to post-test. Results are shown in Table 3.

Cross-tabulations and $t$-tests were run to examine any differences at pre-test between those who completed the study versus those who did not. There were no differences in demographic characteristics, attitudes toward exercise and app use, social support for exercise and app use, 
perceived behavioral control and app use or intention to exercise with fitness apps. People who dropped out of the study had more positive perceptions of exercise, higher frequency of exercise and greater intention to exercise at baseline compared to those who completed the study.

\section{Discussion}

The purpose of this pilot study was to develop and evaluate a fitness app using TPB in order to determine feasibility of incorporating theory constructs into a fitness app. Theory constructs were measured to determine any changes over the eight-week study. To our knowledge, the app developed for this study is the first theory-based app developed to assess exercise behavior change. This is also the first study to test the efficacy of an exercise behavior change app developed using TPB by mapping each theory construct to app features. While it was originally hypothesized that the version of the app with all constructs available to the user (in the form of app features) would be most likely to impact behavior change, the small sample size after attrition and the analysis related to user exercise behaviors before participating in study, led our conclusions to not directly link to the original hypothesis. That said, the theory-based app developed for this study did evoke change in behavioral intention regarding using a fitness app, even though this did not translate to reported increases in exercise frequency. It is interesting to note that the participants who did not complete the study demonstrated greater frequency of exercise prior to the study as well as intentions to exercise. While the researchers cannot say why participants dropped out of the study, it is possible the reason may be related to this, such as participants already had exercise habits and were motivated to carry them out regardless of the study app or perhaps were not further benefiting from the app. While there are studies on how fitness apps influence exercise behavior change, less is known about sustained use of apps after a behavior has become normalized in a person's routine, or whether apps are needed at that point. This is an area worthy of future exploration.

There is much room for improvement in fitness app development, such as translating intention to behavior, testing other health behavior theories, and determining the best ways to develop app features to represent theoretical constructs. One major issue in app development is using behavior change techniques instead of full behavior change theories. Theories are designed to be used with constructs working together to create behavior change. Given the mixed results of studies looking at behavior change techniques instead of full theories $(11,12,22)$, apps should link constructs to create behavior change. As suggested in other disciplines, apps should be developed with the input of the professionals in that discipline (23). This collaboration would help make progress in offering clients and patients helpful apps in their behavior change efforts. A further addition would be to develop apps that incorporate feedback and monitoring from health professionals, which may increase behavior change adherence than the app alone (6). Lastly, the only app feature that cued the user to action was the notification to engage in movement. Otherwise, the user needed to use the app manually. This could be an area of future research to determine an appropriate level of automation versus user initiation.

\section{Limitations}

In this pilot study, there was a small sample size, for two main reasons that are commonly seen in longitudinal app studies. First, there were challenges with recruitment that researchers speculated, such as people were not interested in using a fitness app, people did not want to commit to an 8-week study, or people had an iPhone instead of an Android device. Second, there were issues with study attrition, which is not uncommon in technology-based research (24). Because of the small sample size, we were unable to explore changes in fitness outcomes across app variations; instead the study demonstrates feasibility in measuring changes in behavior, perceptions and intentions. Future research with larger samples is needed to explore changes in fitness outcomes based on the type of theory constructs a participant receives in a fitness app, and if the number or types of constructs received influence the outcome. While this was the initial intent of the present study, due to difficulties with study enrollment and retention we were not able to answer this question. Despite this limitation, the study offers useful information about feasibility of assessing theory-based apps and highlights the importance of continuing to examine how different types of apps based on theory impact health outcomes. Another limitation is that participants self-selected to participate in this study. Therefore, there may be bias, such as already favorable views of being physically active or using fitness apps. In addition, the users were not involved in the development of the app, they only tested the app once 
designed.

Regarding the theory, though the research team made every effort to connect theory constructs to actual app features, there is a chance that the app features did not fully address the construct it intended to address (attitude, subjective norms, perceived behavior control). There is also the chance that TPB was not an ideal theory to use in development of a fitness app.

\section{Future directions}

There are a multitude of future directions for theorybased fitness app research. This same study could be replicated using a larger sample, especially to examine app feature usage. There could also be a comparison of clinical versus non-clinical sample populations. Different health behavior theories could also be used in the development of fitness apps. More research is pointing to the usefulness of including the user in the app design process (25-27). This user involvement in app design combined with health behavior change theory usage could be helpful in eliciting health behavior change. That said, the TPB constructs mapped to the app features in this study are an excellent starting point for future studies.

\section{Acknowledgments}

Funding: None.

\section{Footnote}

Conflicts of Interest: Both authors have completed the ICMJE uniform disclosure form (available at http://dx.doi. org/10.21037/ht-20-17). The authors have no conflicts of interest to declare.

Ethical Statement: The authors are accountable for all aspects of the work in ensuring that questions related to the accuracy or integrity of any part of the work are appropriately investigated and resolved.

Open Access Statement: This is an Open Access article distributed in accordance with the Creative Commons Attribution-NonCommercial-NoDerivs 4.0 International License (CC BY-NC-ND 4.0), which permits the noncommercial replication and distribution of the article with the strict proviso that no changes or edits are made and the original work is properly cited (including links to both the formal publication through the relevant DOI and the license). See: https://creativecommons.org/licenses/by-nc-nd/4.0/.

\section{References}

1. Dennison L, Morrison L, Conway G, et al. Opportunities and challenges for smartphone applications in supporting health behavior change: qualitative study. J Med Internet Res 2013;15:e86.

2. Sama PR, Eapen ZJ, Weinfurt KP, et al. An evaluation of mobile health application tools. JMIR Mhealth Uhealth 2014;2:e19.

3. Connelly J, Kirk A, Masthoff J, et al. The use of technology to promote physical activity in Type 2 diabetes management: a systematic review. Diabetic Med 2013;30:1420-32.

4. Herrmann LK, Kim J. The fitness of apps: a theory-based examination of mobile fitness app usage over 5 months. mHealth 2017;3:2.

5. Cowan LT, Can Wagenen SA, Brown BA, et al. Apps of steel: are exercise apps providing consumers with realistic expectations? a content analysis of exercise apps for presence of behavior change theory. Health Educ Behav 2013;40:133-9.

6. Brunstein A, Brunstein J, Mansar SL. Integrating health theories in health and fitness applications for sustained behavior change: Current state of the art. Creative Educ 2012;3:1-5.

7. Payne HE, Lister C, West JH, et al. Behavioral functionality of mobile apps in health interventions: a systematic review of the literature. JMIR Mhealth Uhealth 2015;3:e20.

8. Azar KM, Lesser LI, Laing BY, et al. Mobile applications for weight management: theory-based content analysis. Am J Prev Med 2013;45:583-9.

9. Conroy DE, Yang CH, Maher JP. Behavior change techniques in top-ranked mobile apps for physical activity. Am J Prev Med 2014;46:649-52.

10. Direito A, Dale LP, Shields E, et al. Do physical activity and dietary smartphone applications incorporate evidencebased behaviour change techniques? BMC Public Health 2014;14:646.

11. Webb TL, Sniehotta FF, Michie S. Using theories of behaviour change to inform interventions for addictive behaviours. Addiction 2010;105:1879.

12. Michie S, Abraham, C, Whittington C, et al. Effective techniques in healthy eating and physical activity interventions: a meta-regression. Health Psychol 
2009;28:690.

13. Glanz K, Rimer B, Viswanath K. Theory, research, and practice in health behavior and health education. 4th ed. San Francisco, CA: Jossey-Bass; 2008.

14. Chatzipavlou IA, Christoforidou SA, Vlachopoulou M. A recommended guideline for the development of $\mathrm{mHealth}$ Apps. mHealth 2016;2:21.

15. Stoyanov SR, Hides L, Kavanagh DJ, et al. Mobile App Rating Scale: A new tool for assessing the quality of health mobile apps. JMIR Mhealth Uhealth 2015;3:e27.

16. Ajzen I. Perceived behavioral control, self-efficacy, locus of control, and the Theory of Planned Behavior. J Appl Soc Psychol 2002;32:665-83.

17. Rhodes RE, Courneya KS, Jones LW. Translating exercise intentions into behavior: personality and social cognitive correlates. J Health Psychol 2003;8:447-58.

18. Ouellette JA, Hessling R, Gibbons FX, et al. Using images to increase exercise behavior: Prototypes versus possible selves. Pers Soc Psychol Bull 2005;31:610-20.

19. Bidargaddi N, Pituch T, Maaieh H, et al. Predicting which type of push notification content motivates users to engage in a self-monitoring app. Prev Med Rep 2018;11:267-73.

20. Poag K, McAuley E. Goal Settings Self-Efficacy, and Exercise Behavior. J Sport Exercise Psy 1992;14:352-60.

doi: $10.21037 /$ ht-20-17

Cite this article as: Herrmann LK, Blackstone SR. Feasibility and outcomes from development and testing of a health behavior theory-based fitness app. Health Technol 2020;4:4.
21. Rabin C, Bock B. Desired features of smartphone applications promoting physical activity. Telemed J E Health 2011;17:801-3.

22. Middelweerd A, Mollee JS, van der Wal CN, et al. Apps to promote physical activity among adults: a review and content analysis. Int J Behav Nutr Phys Act 2014;11:97.

23. Chen J, Lieffers J, Bauman A, et al. Designing health apps to support dietetic professional practice and their patients: Qualitative results from an international survey. JMIR Mhealth Uhealth 2017;5:e40.

24. Eysenbach G. The law of attrition. J Med Internet Res 2005;7:e11.

25. Eyles H, Jull A, Dobson R, et al. Co-design of mHealth delivered interventions: A systematic review to assess key methods and processes. Curr Nutr Rep 2016;5:160-7.

26. Verbiest MEA, Corrigan C, Dalhousie S, et al. Using codesign to develop a culturally tailored, behavior change mHealth intervention for indigenous and other priority communities: A case study in New Zealand. Transl Behav Med 2019;9:720-36.

27. Jennings HM, Morrison J, Akter K, et al. Developing a theory-driven contextually relevant $\mathrm{mHealth}$ intervention. Glob Health Action 2019;12:1550736. 